\title{
HIV self-screening distribution preferences and experiences among men who have sex with men in Mpumalanga Province: Informing policy for South Africa
}

\author{
O Radebe, ${ }^{1,2} \mathrm{MB} \mathrm{ChB}$; S A Lippman, ${ }^{2} \mathrm{PhD}, \mathrm{MPH} ; \mathrm{T}$ Lane, ${ }^{2,3} \mathrm{PhD}$; H Gilmore, ${ }^{2} \mathrm{MPH}$; E Agnew, ${ }^{2} \mathrm{PhD} ;$ A Manyuchi, ${ }^{1} \mathrm{PhD}$; \\ J A McIntyre, ${ }^{1,4} \mathrm{MB} \mathrm{ChB}, \mathrm{FRCOG}$ \\ ${ }^{1}$ Anova Health Institute, Johannesburg, South Africa \\ ${ }^{2}$ Center for AIDS Prevention Studies, Division of Prevention Science, University of California, San Francisco, CA, USA \\ ${ }^{3}$ Equal International, Horsham, UK \\ ${ }^{4}$ School of Public Health and Family Medicine, Faculty of Health Sciences, University of Cape Town, South Africa
}

Corresponding author: O Radebe (radebelebo11@gmail.com)

\begin{abstract}
Current research suggests that HIV self-screening (HIVSS) is a feasible and acceptable approach to increase HIV testing among men who have sex with men (MSM). However, few data are available to shape policy around dissemination and implementation. Gaps in knowledge include preferences for distribution of HIVSS kits, potential social harms and benefits of their use, and how much test users would be willing to pay for the kits. The aim was to inform policy recommendations to optimise distribution of HIVSS kits to MSM in South Africa (SA), where there is a high HIV incidence and unmet testing needs. MSM in the high-HIV-prevalence Gert Sibande and Ehlanzeni districts of Mpumalanga Province, SA, were enrolled between October 2015 and May 2017. Participants were provided with their choice of blood or oral fluid HIVSS test kits, receiving 5 kits at enrolment and 4 additional kits at the 3-month follow-up visit. Questionnaires were administered at enrolment, 3 months and 6 months. We analysed participants' reported social benefits and harms, and their preferences for kit distribution and pricing. Among 127 MSM screened and enrolled, 114 responded to follow-up questionnaires regarding distribution preferences, $49.3 \%$ preferred to acquire HIVSS kits at a community-based organisation (CBO) and $42.7 \%$ at a clinic, with $8 \%$ preferring a pharmacy. Participants with higher education preferred CBO sites for distribution; in other respects preferences were similar by demographic characteristics. Reported social benefits were common, including knowing one's status, prevention knowledge gained and improved communication with partners. Despite ubiquitous interest in using the kits, the majority of MSM could not afford to purchase test kits. SA guidelines have integrated HIVSS into HIV and testing policy, but little has been published regarding distribution channels of the kits for MSM and other key populations. There is a partnership between the National Department of Health and CBOs that specialise in key population programming to ensure MSM and other populations with unmet testing needs can access affordable test kits. We observed no social harms, and there were multiple social benefits. Consequently, we recommend immediate free or lowcost distribution of HIVSS kits to MSM through community-based initiatives. Future research should continue to assess optimised linkage to care.
\end{abstract}

S Afr Med J 2019;109(4):227-231. DOI:10.7196/SAMJ.2019.v109i4.13818

South Africa (SA) has the largest HIV epidemic in the world; therefore, it is critical to expand HIV testing services and early initiation of antiretroviral treatment to reduce AIDS mortality and onward transmission. ${ }^{[1]}$ Too few South Africans know their HIV status, with a large proportion of people living with HIV undiagnosed. ${ }^{[2]}$ In SA, despite great progress towards the Joint United Nations Programme on HIV and AIDS (UNAIDS) 90-90-90 targets, which were 85\%-71\%-87\% in 2017, the HIV prevalence remains high in certain provinces, e.g. Mpumalanga Province, SA, with an increase of $14-17.3 \%$ in 2012 - 2017.[2,3] The National Department of Health devised strategies to expand the HIV testing service (HTS), but poor uptake of testing and failure to link to and remain in care persist, leading to late diagnosis and increased morbidity and mortality. ${ }^{[3]}$ The failure to test and link to care is more pronounced among men who have sex with men (MSM) than the general population. While an estimated $40 \%$ of all HIVpositive men are unaware of their HIV status, estimates among MSM are currently closer to $70 \%{ }^{[4,5]}$ Current HTS coverage is insufficient among hard-to-reach groups, such as MSM and sex workers, who bear a disproportionate burden of HIV and experience barriers to accessing HIV testing in clinics. Research suggests that increased coverage of clinic-based HTSs is insufficient to encourage improved testing uptake and treatment initiation, and therefore does not reduce rates of HIV infection in high-risk groups. ${ }^{[6]}$

It is critical that we expand HIV testing options for key populations with approaches that are responsive to their needs. HIV self-screening (HIVSS) has been found to be highly acceptable among MSM, ${ }^{[7-21]}$ and may be effective in reducing the time to diagnosis and treatment and thus the incidence of new HIV infections. ${ }^{[9]}$ MSM at our research site in Mpumalanga preferred HIVSS to clinic-based testing, with $>80 \%$ stating a preference for the former. ${ }^{[7]}$ Despite its potential, HIVSS has not been fully embraced by policymakers. This is partly due to lack of information regarding how best to deliver or distribute tests and how to financially structure the cost of distributing HIVSS nationally. ${ }^{[10]}$ Fear of social harms, including psychological risk and potential coercion of stigmatised populations, has also delayed self-screening expansion.

Policy regarding HIVSS and the legal framework to include it in national HTS efforts vary according to country. ${ }^{[1]}$ High-income countries, such as the USA and UK, introduced over-the-counter (OTC) sales in early 2012 and 2014, respectively. France and Ireland subsequently implemented their HIVSS policies. Among African countries, Kenya was the first to develop its HTS national policy to include the oral fluid HIVSS kit in 2008. ${ }^{[12]}$ In SA, before 2015, 
unregulated sale of HIVSS kits in the absence of policy raised concerns of misuse of OTC sales by retail pharmacies and supermarkets. ${ }^{[13]}$ In May 2015, the SA Pharmacy Council facilitated legalising the selling of HIVSS kits by adding them to the pharmacy register. This followed the lifting of a 1-year ban of the kits by the Pharmacy Council and marked a major milestone towards adopting HIVSS in SA. The Southern African HIV Clinicians Society developed a document on self-testing policy and guidance considerations. ${ }^{[14]}$ This was followed by the publication of the National Department of Health HIVSS guidelines in May 2018, ${ }^{[22]}$ which focus on considerations for effective and safe use of HIVSS. While potential distribution channels are listed (communitybased, facilities-based, secondary distribution to partners), detailed guidance for implementation (when, where and how to distribute tests) is not provided, given the lack of data across target groups needed to guide implementation. Key populations, with large unmet needs for accessible tests, should be prioritised regarding HIVSS roll-out, which requires targeted distribution. Although the healthcare system in SA can be accessed through local clinics, clinic-based testing is often impractical for MSM, who face barriers and stigma related to public health services owing to their sexual orientation. The SA government will possibly need to consider other distribution channels, such as community-based organisations (CBOs) and peer groups, which may be more readily accessible to key populations. ${ }^{[13]}$

The inclusion of HIVSS as part of the SA HTS policy could contribute to testing uptake and frequency, moving the country closer to its target of $90-90-90$ by $2020 .{ }^{[15]}$ To achieve these targets, gaps in programming for the needs of MSM must be addressed. We aimed to provide data directly applicable to inform policy, which would enable widespread distribution and use of HIVSS by MSM in SA, thus specifically addressing the current gaps in knowledge regarding where and how these test kits can be distributed, and identifying potential social benefits and harms linked to HIVSS.

\section{Methods}

This study was conducted among MSM at two study sites in periurban districts of Mpumalanga: Gert Sibande, semi-rural, but with a vibrant gay-identified community of MSM, whose networks span the rural towns of Msukaligwa (Ermelo), Makhado (Piet Retief), Secunda, Standerton and Volksrust; and Ehlanzeni district municipality, which contains the administrative capital Mbombela (Nelspruit) - a hub for tourists visiting the Kruger National Park. These two sites were home to the Mpumalanga Men's Study (MPMS) (2012 - 2015), ${ }^{[3]}$ a series of crosssectional seroprevalence surveys using respondent-driven sampling (RDS), as well as Project Boithato, an adapted Mpowerment Project focusing on MSM. Participants were randomly selected among those not known to be HIV-positive from the most recent MPMS RDS survey in Gert Sibande. In Ehlanzeni, participants were recruited from the most recent MPMS survey and, as more time had elapsed and fewer MPMS participants were reachable, participants were recruited with a new RDS. ${ }^{[7]}$ Criteria for the RDS surveys included being $\geq 18$ years of age and sexually active (oral or anal intercourse) with at least one other man in the past 6 months. The recruitment, eligibility procedures and outcomes for MPMS and the main HIVSS study are described elsewhere. ${ }^{[3,7]}$

\section{Data collection}

Participants were invited to the study sites, which are located in both areas at the offices of a local non-governmental organisation known in the community for working with MSM. Participants provided written informed consent and, if interested in participating, were then tested for HIV by a trained test counsellor to ensure negative status prior to enrolment. Participants completed a behavioural questionnaire and were shown and provided instructions and a demonstration of use of blood (AtomoRapid HIV-1/2 Antibody Test, Atomo Diagnostics, Australia) and oral fluid-based (OraQuick ADVANCE Rapid HIV-1/2 Antibody Test, OraSure Technologies, USA) self-test kits. They were provided with up to 5 HIVSS kits - either blood or oral fluid based, depending on their choice - and asked to use these at least once before a subsequent visit after 3 months. Participants could share the tests with sexual partners or others with whom they felt comfortable.

Participants returned for a follow-up visit 3 months after enrolment, when they responded to a questionnaire regarding use of the kits, preferences for use and social harms and benefits. They were offered an additional 4 HIVSS kits of their choice (either blood or oral fluid based, which could be different from their choice at baseline) and asked to return 6 months after enrolment for a final follow-up visit, which included a questionnaire with the acceptability/feasibility and social harms/benefits measures described above, as well as items regarding participants' experiences with self-screening and preferences for distribution and pricing.

If participants were to obtain test kits in the future, they were asked where they would prefer to collect them. Response options included: CBOs, clinics, pharmacies or another distribution site. Only 1 participant opted for something other than these responses, stating 'anywhere they are available. Participants were asked how much they would be willing to pay for test kits in local SA currency - this was an open-ended rather than multiple-choice response. Response options were later grouped into: would not pay for HIVSS kits; would pay $<$ ZAR24 $(<\mathrm{USD} 2)$; or $>$ ZAR25 but not $>$ ZAR49; or $>$ ZAR50 (>USD4). Participants were also asked about social benefits and harms. One of the questions enquired if anything bad had happened as a result of participating in the study, and another if anything good had happened. These open-ended responses were qualitatively coded into one or more themes, including the primary good and bad outcomes for each participant. Participants requiring medical follow-up (testing positive or reporting other clinical needs) were referred to local MSMcompetent healthcare facilities.

\section{Data analysis}

Data analysed included responses to the behavioural questionnaires at baseline demographics, and to the acceptability and feasibility questionnaires at 3 and 6 months. Data were captured using the Questionnaire Development Software (QDS) (NOVA Research, USA) computer-assisted personal interview (CAPI) module, and exported to Stata 14 (StataCorp., USA). We generated basic frequency tables to describe the population characteristics. We also assessed HIVSS distribution preferences by demographic characteristics and used Fisher's exact test to assess these differences. We report distribution of reported benefits and harms, and willingness to pay for HIVSS.

\section{Ethical approval}

Ethical approval to conduct the study was obtained from the University of California, San Francisco Committee on Human Research (ref. nos iIRB14-13678 and 167347) and the University of the Witwatersrand Human Research Ethics Committee (ref. no. M140725). The US Centers for Disease Control and Prevention, one of the study sponsors, also approved the protocol, as well as the Mpumalanga Department of Health and Social Development Research Committee.

\section{Resullts}

\section{Demographic characteristics}

Of 127 eligible participants who consented to and enrolled in the study at baseline, 51 from Gert Sibande and 63 from Ehlanzeni ( $N=114$ ) responded to questionnaire items regarding their experiences and preferences with an HIVSS kit at follow-up, including cost and 
preference for collection, and are included in the analysis. They were notably young, with $65 \%<25$ years of age, and only one-third employed in the past 6 months. Other demographic results are described elsewhere. ${ }^{[7]}$ Table 1 includes preferences for HIVSS distribution by demographic characteristics.

Preference for distribution point was similar across demographic characteristics, with the exception of those with some college or technical education (92\%), who preferred the CBO option to the clinic or pharmacy option $(p<0.01)$. Younger men (18 - 24 years old), and those who did not identify as gay or bisexual, demonstrated some preference for distribution at community organisations (49.4\%) and clinics (46.8\%) rather than at a pharmacy (3.9\%). Participants in Gert Sibande also favoured $\mathrm{CBO}$ distribution points $(>60 \%)$.

\section{Social benefits and harms}

At the 3-month visit, the majority of participants at both study sites - 97.9\% in Gert Sibande and 94\% in Ehlanzeni - reported only social benefits, or some 'good thing' that had happened to them as a result of being in the HIVSS study. At 6 months, $100 \%$ at both sites who returned for their follow-up visit reported benefits, and only $2.1 \%$ reported any harm. The responses largely mapped into 6 categories of benefits and 2 categories of harms. The most commonly reported benefit was knowledge gain of HIV and prevention, which was noted by $\sim 30 \%$ of those in Gert Sibande at the 3-and 6-month visits and by $>50 \%$ of the participants at 3 months (54\%) and at 6 months (68\%) in Ehlanzeni (Table 2). The benefit of knowledge of HIV status was also often reported at both sites by $\sim 35 \%$ of participants at 3 months. Improved communication with partners was reported by $25 \%$ and $44 \%$ of those in Gert Sibande at 3 and 6 months, respectively, although this was less common in Ehlanzeni - reaching 18.5\% at 6 months. The most common secondary themes of benefits emerged as privacy, empowerment gained from testing oneself and other community benefits (e.g. educating family and friends about HIVSS kits).

Reported harms were rare. At the Gert Sibande study site, 1 person reported experiencing social pressure to distribute kits due to high demand in the community. There were no social harms reported at the 6-month follow-up visits. Two participants in Ehlanzeni noted that receiving information relating to HIV-seropositive test results from friends, family or partners to whom they distributed kits, was a negative experience.

\section{HIV self-screening kit pricing preference}

Overall, 44 participants (38.6\%) stated that they would not pay for an HIVSS test, given access to free testing at the clinic (Table 3). Less than one-quarter would pay $<$ ZAR24 $(<\mathrm{USD} 2)$ and $<24 \%$ would pay ZAR25 - $49(<\mathrm{USD} 4)$ if the kits were available for purchase or sold OTC. A small proportion (13.2\%) of participants were willing to pay >ZAR50 ( USD4) for the HIVSS kits if these were available commercially. Willingness to pay did not differ substantively by age, sexual identity or testing history; those with tertiary education were a little less willing to pay for HIVSS than those with Matric (secondary school) only.

Table 1. Preference for HIV self-test distribution by demographic characteristics of the HIVSS MSM cohort

\begin{tabular}{|c|c|c|c|c|}
\hline \multirow[b]{2}{*}{ Characteristics } & \multicolumn{4}{|c|}{ Preference for HIVSS distribution point $(N=114)$} \\
\hline & $\begin{array}{l}\text { Community organisation } \\
(n=58,50.9 \%), n(\%)\end{array}$ & $\begin{array}{l}\text { Clinic } \\
(n=50,43.9 \%), n(\%)\end{array}$ & $\begin{array}{l}\text { Pharmacy } \\
(n=6,5.3 \%), n(\%)\end{array}$ & $\begin{array}{l}\text { Fisher's exact } \\
p \text {-value }\end{array}$ \\
\hline Age, years & & & & 0.21 \\
\hline $18-24$ & $37(49.3)$ & $32(42.7)$ & $6(8.0)$ & \\
\hline $25-39$ & $21(53.8)$ & $18(46.2)$ & $0(0.0)$ & \\
\hline Education & & & & $<0.01$ \\
\hline Primary or secondary & $27(49.1)$ & $27(49.1)$ & $1(1.8)$ & \\
\hline Matric (graduated high school) & $19(41.3)$ & $23(50.0)$ & $4(8.7)$ & \\
\hline Some college or technical school & $12(92.3)$ & $0(0.0)$ & $1(7.7)$ & \\
\hline Employment in past 6 months & & & & 0.23 \\
\hline Yes & $21(58.3)$ & $12(33.3)$ & $3(8.3)$ & \\
\hline No & $37(47.4)$ & $38(48.7)$ & $3(3.8)$ & \\
\hline Sexual identity & & & & 0.71 \\
\hline Gay/homosexual & $17(51.5)$ & $13(39.4)$ & $3(9.1)$ & \\
\hline Bisexual & $38(49.4)$ & $36(46.8)$ & $3(3.9)$ & \\
\hline Straight & $2(66.7)$ & $1(33.3)$ & 0 & \\
\hline Transgender & $1(100)$ & 0 & 0 & \\
\hline Sexual partners in past 6 months & & & & 0.53 \\
\hline 0 & $2(66.7)$ & $1(33.3)$ & 0 & \\
\hline 1 & $38(55.9)$ & $36(38.2)$ & $4(5.9)$ & \\
\hline$\geq 2$ & $18(41.9)$ & $23(53.5)$ & $2(4.7)$ & \\
\hline Testing history prior to study, months & & & & 0.85 \\
\hline $0-6$ & $21(45.7)$ & $21(45.7)$ & $4(8.7)$ & \\
\hline$>6-12$ & $17(54.8)$ & $12(38.7)$ & $2(6.5)$ & \\
\hline$>12$ & $10(52.6)$ & $9(47.4)$ & 0 & \\
\hline Never tested & $10(55.6)$ & $8(44.4)$ & 0 & \\
\hline Study site location & & & & 0.17 \\
\hline Gert Sibande & $31(60.8)$ & $18(35.3)$ & $2(3.9)$ & \\
\hline Ehlanzeni & $27(42.9)$ & $32(50.8)$ & $4(6.3)$ & \\
\hline
\end{tabular}




\section{Discussion}

Our study demonstrates a clear preference among MSM in these two rural districts for distribution of HIVSS kits through CBOs that specialise in key population programming. It also shows that clinic-based distribution is feasible and acceptable to many MSM (or to MSM who are openly 'out' regarding their gay-identified sexual orientation) once the HIVSS kits become available. In the SA context, policymakers frequently support centralisation of services in major hospitals, clinics or pharmacies; our data suggest that centralised distribution through the public health service may hinder optimal uptake of HIVSS among MSM, who still face stigma and discrimination in accessing healthcare services, particularly in seeking HIV and other sexual healthcare..$^{[8]}$ Bisexual and other non-gay identified MSM, who are the most 'hidden' members of the MSM population, may fear having their sexual practices disclosed by presenting at clinics for HIV testing as MSM; it is critical to increase uptake of HIV testing among these men, who, as primarily insertive partners with men, may acquire and transmit HIV through either homosexual and heterosexual intercourse. ${ }^{[16]}$ The preference for distribution through CBOs may allow HIVSS to be a complementary service while the organisations engage in peer-led outreach activities. Based on these research findings, key and hidden populations will possibly have improved access to and uptake of HIVSS kits if distributed through CBOs.

Our research found no evidence of social harms related to HIVSS among MSM other than the increasing demand for kits in these communities and learning of the seropositive results of others to whom MSM had distributed kits. Instead, findings indicated that in this MSM community, exposure to HIVSS elicited the value placed on knowing one's status and the eagerness of the community to test more frequently and acquire more knowledge. Empowerment was also cited as a social benefit resulting from HIVSS, emphasising the power of autonomy and taking pride and responsibility in regular testing. Finally, $25 \%$ of MSM in our cohort reported the benefit of increased testing discussions and communication with partners, which signalled the potential for HIVSS to encourage disclosure discussions. Couple counselling and testing is uncommon, even among heterosexuals; it might even be less common among MSM who do not access clinic services. ${ }^{[17]}$ Other studies have shown that among gay couples, an existing dynamic of support and communication around HIV influences sexual risk behaviour in relationships outside the primary relationship, leading to decreased risk of HIV transmission. ${ }^{[18]}$ This open communication not only encourages regular HIV testing, but influences more informed choices regarding sexual practices among MSM. HIVSS provided an opportunity for regular testing and HIV support, which may involve disclosure if one should test HIV-positive. ${ }^{[19]}$ In our study, testing with partners or friends had an empowering effect on MSM participants, not only regarding HIV support, but also by indicating the potential of uptake of HIV testing in the general population, where some participants distributed test kits to non-MSM family members.

Policymakers have had concerns regarding HIVSS possibly eliminating the role of pre- and post-test counselling, which has been the cornerstone of the SA HIV programme. Concerns about psychological distress among those who test in the absence of professional counselling were unfounded in this cohort. None of the 114 participants mentioned lack of counselling as a problem. As there is a preference for the distribution of kits through $\mathrm{CBOs}$, this provides a window of opportunity to train peer outreach workers, as well as

Table 2. Reported social benefits and harms to participation in HIV self-screening

\begin{tabular}{|c|c|c|c|c|}
\hline \multirow[b]{2}{*}{ Benefits and harms ${ }^{*}$} & \multicolumn{2}{|c|}{ Gert Sibande } & \multicolumn{2}{|c|}{ Ehlanzeni } \\
\hline & $\begin{array}{l}\text { months } \\
(n=48), n(\%)\end{array}$ & $\begin{array}{l}6 \text { months } \\
(n=45), n(\%)\end{array}$ & $\begin{array}{l}\text { months } \\
(n=50), n(\%)\end{array}$ & $\begin{array}{l}6 \text { months } \\
(n=65), n(\%)\end{array}$ \\
\hline \multicolumn{5}{|l|}{ Social benefits } \\
\hline Knowledge gained regarding HIV/prevention & $15(31.3)$ & $13(28.9)$ & $27(54.0)$ & $44(67.7)$ \\
\hline Knowing HIV status & $17(35.4)$ & $11(24.4)$ & $18(36.0)$ & $12(18.5)$ \\
\hline Improved partner communication & $12(25.0)$ & $20(44.4)$ & $3(6.0)$ & $12(18.5)$ \\
\hline Privacy & $3(6.3)$ & $1(2.2)$ & $3(6.0)$ & - \\
\hline Empowerment & $8(16.7)$ & $3(6.7)$ & $6(12.0)$ & $11(16.9)$ \\
\hline Benefits to others/community & $9(18.8)$ & $1(2.2)$ & $8(16.0)$ & - \\
\hline Reported no benefit & $1(2.1)$ & - & $2(4.0)$ & $4(6.2)$ \\
\hline \multicolumn{5}{|l|}{ Social harms } \\
\hline Pressure to distribute kits & $1(2.1)$ & - & - & - \\
\hline Testing positive and receiving results & - & - & $2(4.0)$ & - \\
\hline Reported no harms & $47(97.9)$ & $45(100.0)$ & $47(94.0)^{\dagger}$ & $65(100.0)$ \\
\hline
\end{tabular}

Table 3. Willingness of MSM cohort to pay for an HIVSS kit at final study visit

\begin{tabular}{llll}
\hline HIVSS kit distribution & $\begin{array}{l}\text { Overall } \\
(\boldsymbol{N = 1 1 4}), \boldsymbol{n}(\%)\end{array}$ & $\begin{array}{l}\text { Gert Sibande } \\
(\boldsymbol{n}=\mathbf{5 1}), \boldsymbol{n}(\%)\end{array}$ & $\begin{array}{l}\text { Ehlanzeni } \\
(\boldsymbol{n}=\mathbf{6 3}), \boldsymbol{n}(\%)\end{array}$ \\
\hline $\begin{array}{l}\text { How much would you pay for the HIVSS kit? } \\
\quad \text { Would not pay }\end{array}$ & $44(38.6)$ & $19(37.3)$ & $25(39.7)$ \\
$\quad$ Would pay <ZAR24 (<USD2) & $28(24.6)$ & $20(39.2)$ & $8(12.7)$ \\
Would pay ZAR25 - 49 (<USD4) & $27(23.7)$ & $10(19.6)$ & $17(27.0)$ \\
Would pay $\geq Z$ ZAR50 ( $\geq$ USD4) & $15(13.2)$ & $2(3.9)$ & $13(20.6)$ \\
MSM = men who have sex with men; HIVSS = HIVself-screening. & & &
\end{tabular}


other community-based counsellors. Thus, they can intervene in cases of harmful psychosocial effects and encourage confirmatory testing, where counselling is provided.

We further note that our findings argue against the exclusive introduction of cost-based HIVSS, suggesting that it may lead to barriers in accessing the test kits in this semi-rural and largely lowincome community, as $<15 \%$ were prepared to pay $>$ ZAR50. With the recent agreement between OraSure Technologies and the Bill and Melinda Gates Foundation, which made the OraQuick HIVSS test available at a reduced price of USD2 in the public sector in 50 countries, including SA, ${ }^{[20]} \sim 25 \%$ of our cohort participants would be able to afford the kits.

\section{Study limitations}

We acknowledge the small sample size, which limits our ability to generalise findings to the entire MSM population of SA. Retention was high (89\%); however, we are unable to report on the attitudes and perceptions of those who did not return, who may be less receptive to HIVSS. Self-reported outcomes are always subject to social desirability and recall bias. Our method did not allow us to directly observe MSM partner testing. Furthermore, some of the lingering questions in the field about linkage, which are important, fell outside of the feasibility/ acceptability scope of the study. Future studies are needed to explore some of these remaining questions.

\section{Conclusion}

Despite the limitations of this small observational study, the overwhelming perception of the benefits of HIVSS was quite clear in the community, where increasing access to HIV testing is imperative. We found evidence that MSM would benefit from accessing HIVSS through non-governmental and/or CBOs working with key populations at no or low cost. There were many reported social benefits of HIVSS and negligible social harms. Much work still needs to be done to investigate best practices for linkage to care for those who test HIVpositive using HIVSS; nonetheless, HIVSS has the potential to increase uptake of testing among hard-to-reach groups, thereby enhancing progress on reaching the first 90 . In SA, to reach each of the 90-90-90 targets, the focus should be on HIVSS, which may be the cornerstone of addressing major barriers to achieving these targets by 2030, thereby reaching those with the largest unmet need for alternatives to HTS.

\section{Declaration. None.}

Acknowledgements. We thank the Anova Health Institute and University of California, San Francisco (UCSF) staff who supported the study: Helen Struthers and Albert Manyuchi (Anova), Tom Osmand (UCSF), and the study team at Ehlanzeni and Gert Sibande for their support with data collection.

Author contributions. OR, SAL, TL and JAM helped with the study design and gave guidance with regard to the study implementation. HG and AM supervised study implementation and data collection. EA contributed to data analysis. All authors contributed to data interpretation and drafting of the manuscript.

Funding. Research reported in this publication was supported by the National Institute of Mental Health of the National Institutes of Health (NIH) (R21MH103038) and US Centers for Disease Control and
Prevention (U2GHH000251). The content is solely the responsibility of the authors and does not necessarily represent the official views of the $\mathrm{NIH}$. We wish to acknowledge support from the UCSF International Traineeships in AIDS Prevention Studies (ITAPS) Program (US NIMH, R25MH064712), and from the CV Starr Foundation Scholarship Fund.

Conflicts of interest. HIV self-screening kits were donated by Atomo Diagnostics and OraSure Technologies; however, test kit manufacturers played no role in study design, data analysis, data interpretation or decision to publish.

1. Johnson LF, Dorrington RE, Moolla H. Progress towards the 2020 targets for HIV diagnosis and antiretroviral treatment in South Africa. South Afr J HIV Med 2017;18(1):1694. https://doi. org/10.4102sajhivmed.v18i1.694

2. Simbayi L, Shisana O, Rehle T, et al. South African National HIV Prevalence, Incidence and Behaviou Survey, 2012. Pretoria: Human Sciences Research Council, 2014:67-75.

3. Lane T, Osmand T, Marr A, et al. The Mpumalanga Men's Study (MPMS): Results of a baseline biological and behavioral HIV surveillance survey in two MSM communities in South Africa. PLOS ONE 2014;9(11):111063. https://doi.org/10.1371/journal.pone.0111063

4. Lane T, Osmand T, Marr A, Struthers H, McIntyre JA, Shade SB. High HIV incidence in a South African community of men who have sex with men: Results from the Mpumalanga Men's Study, 2012 - 2015. community of men who have sex with men: Results from the Mpumalanga Men's Study, 2012 - 20
J Acquir Immune Defic Syndr 2016;73(5):609-611. https://doi.org/10.1097qai.0000000000001162

5. Kamali A, Price MA, Lakhi S, et al. Creating an African HIV clinical research and prevention trials network: HIV prevalence, incidence and transmission. PLOS ONE 2015;10(1):0116100. https://doi org/10.1371journal.pone. 0116100

6. Makusha T, Knight L, Taegtmeyer M, et al. HIV self-testing could 'revolutionize testing in South Africa, but it has got to be done properly': Perceptions of key stakeholders. PLOS ONE 2015;10(3):0122783. https://doi.org/10.1371journal.pone.0122783

7. Lippman SA, Lane T, Rabede O, et al. High acceptability and increased HIV-testing frequency after introduction of HIV self-testing and network distribution among South African MSM. J Acquir introduction of HIV self-testing and network distribution among South African MSM.
Immune Defic Syndr 2018;77(3):279-287. https://doi.org/10.1097qai.0000000000001601

8. Volk JE, Lippman SA, Grinsztejn B, et al. Acceptability and feasibility of HIV self-testing among men who have sex with men in Peru and Brazil. Int J STD AIDS 2015;27(7):531-536. https://doi. org/10.1177/0956462415586676

9. Figueroa C, Johnson C, Verster A, Baggaley R. Attitudes and acceptability on HIV self-testing among ke populations: A literature review. AIDS Behav 2015;19(11):1949-1965. https://doi.org/10.1007/s10461 015-1097-8

10. Gomez A, Rosenberg T, Stankard P, et al. Using research to inform the development of social marketing campaigns for HIV self-testing in Kenya and South Africa. https://www.psi.org/wp-content/ uploads/2015/04/Social-Marketing-Campaign.pdf (accessed 14 February 2019).

11. Mavedzenge SN, Baggaley R, Corbett EL. A review of self-testing for HIV: Research and policy Mavedzenge SN, Baggaley R, Corbett EL. A review of self-testing for HIV: Research and policy
priorities in a new era of HIV prevention. Clin Infect Dis 2013;57(1):126-138. https://doi.org/10.1093/ cid/cit156

12. Wong V, Johnson C, Cowan E, et al. HIV self-testing in resource-limited settings: Regulatory and policy considerations. AIDS Behav 2014;18(S4):415-421. https://doi.org/10.1007s10461-014-0825-9

13. Richter M, Venter W, Gray A. Home self-testing for HIV: AIDS exceptionalism gone wrong. S Afr Med 2010;100(10):636-642. https://doi.org/10.7196samj.4198

14. Venter F, Majam M, Jankelowitz L, et al. South African HIV self-testing policy and guidance considerations. South Afr J HIV Med 2017;18(1):775. https://doi.org/10.4102sajhivmed.v18i1.775

15. Williams BG, Gouws E, Somse P, et al. Epidemiological trends for HIV in southern Africa: Implications for reaching the elimination targets. Curr HIV/AIDS Rep 2015;12(2):196-206. https:// doi.org/10.1007s11904-015-0264-x

16. Choko AT, MacPherson P, Webb EL, et al. Uptake, accuracy, safety, and linkage into care over two year Choko AT, MacPherson P, Webb EL, et al. Uptake, accuracy, safety, and linkage into care over two years
of promoting annual self-testing for HIV in Blantyre, Malawi: A community-based prospective study. PLOS Med 2015;12(9):1001873. https://doi.org/10.1371journal.pmed.1001873

17. World Health Organization. Guidance on Couples HIV Testing and Counselling Including Antiretroviral Therapy for Treatment and Prevention in Serodiscordant Couples: Recommendation for a Public Health Approach. Geneva: WHO, 2012.

18. Darbes LA, Chakravarty D, Beougher SC, Neilands TB, Hoff CC. Partner-provided social support influences choice of risk reduction strategies in gay male couples. AIDS Behav 2012;16(1):159-167. https://doi.org/10.1007s10461-010-9868-8

19. Lippman SA, Moran L, Sevelius J, et al. Acceptability and feasibility of HIV self-testing among transgender women in San Francisco: A mixed methods pilot study. AIDS Behav 2016;20(4):928-938. https://doi.org/10.1007s10461-015-1236-2

20. OraSure Technologies. OraSure partners with the Bill \& Melinda Gates Foundation to drive accelerated adoption of OraQuick HIV self-testing. https://globenewswire.com/news-release/2017/06/27/1029393/0/ en/OraSure-Technologies-to-Drive-Accelerated-Adoption-of-OraQuick-HIV-Self-Test.html (accessed 14 February 2019).

21. Lippman SA, Gilmore HJ, Lane T, et al. Ability to use oral fluid and fingerstick HIV self-testing (HIVST) among South African MSM. PLOS ONE 2018;13(11):0206849. https://doi.org/10.1371/ journal.pone. 0206849

22. National Department of Health. National HIV Self Screening Guidelines. Pretoria: NDoH, 2018.

23. Human Sciences Research Council. The Fifth South African National HIV Prevalence, Incidence, Behaviour and Communication Survey, 2017: HIV Impact Assessment Summary Report. Cape Town: HSRC, 2018.

Accepted 22 January 2019 